\title{
Um Processo para o Gerenciamento de Riscos em
}

\section{Projetos de Software}

\author{
Pascale Correia Rocha Pereira ${ }^{1}$, Fca. Márcia G. S. Gonçalves ${ }^{1,2}$, \\ Carlo Giovano Pires ${ }^{2}$, Arnaldo Dias Belchior ${ }^{1}$ \\ ${ }^{1}$ Mestrado em Informática Aplicada, Universidade de Fortaleza \\ Av. Washington Soares 1321, 60811-341 - Fortaleza - CE, Brasil \\ ${ }^{2}$ Instituto Atlântico - SPD - Suporte ao Processo de Desenvolvimento \\ Rua Chico Lemos, 946 - Cidade dos Funcionários - CEP 60.822 -780 \\ Fone 55 (85) 3216- 7800 - Fortaleza - CE - Brasil \\ \{pascale.mia, belchior\}@unifor.br, \{marcia, cgiovano\}@atlantico.com.br
}

\begin{abstract}
Risk Management has become more notable in software development projects, as uncertainty is inherent to this kind of projects. Risk management leads the organization to systematically plan, anticipate, and mitigate risks to proactively eliminate or minimize their impact on the project. This paper proposes a software risk management process based on concepts of the RUP, on risk approaches of CMMI-SW, PMBOK, IEEE STD 1540 and AS/NZS 4360. After that, it presents the process matching and implementing in an organization, aiming to follow CMMI level 3 requirements for risk management.
\end{abstract}

Resumo. O gerenciamento de riscos está se tornando cada vez mais relevante em projetos de desenvolvimento de software, devido ao alto grau de incerteza envolvido neste tipo de projeto. O gerenciamento de riscos conduz a organização à sistematicamente planejar, antecipar e mitigar os riscos para proativamente eliminar ou minimizar seus impactos nos projetos. Este trabalho propõe um processo para o gerenciamento de riscos de projetos de software fundamentado em conceitos do RUP, nas abordagens de risco do CMMI-SW, do PMBOK, do padrão IEEE STD 1540 e da AS/NZS 4360. Em seguida, apresenta a sua especialização e implantação em uma empresa, visando atender os requisitos de gerenciamento de riscos do CMMI-SW nível 3.

\section{Introdução}

O gerenciamento de riscos trabalha justamente com a incerteza, visando a identificação de problemas e oportunidades potenciais antes que ocorram, com o objetivo de eliminar ou reduzir a probabilidade de ocorrência e o impacto de eventos negativos para os objetivos do projeto, além de potencializar os efeitos da ocorrência de eventos positivos.

Através da experiência, observou-se que os projetos que empregavam o gerenciamento de riscos tendiam a produzir melhores resultados do que aqueles que não empregavam. Isto decorre do fato de que as coisas raramente ocorrem conforme o planejado, e o gerenciamento de riscos é justamente uma forma melhor de lidar com os riscos do que ficar esperando passivamente que eles se materializem e ataquem o projeto. Alguns autores 
consideram o gerenciamento de riscos uma das atividades mais importantes do gerenciamento de projetos [Brown, 1996; Sommerville, 2003].

O gerenciamento de riscos é abordado por vários modelos dentre os quais o PMBOK (2004), o CMMI-SW (2002), a AS/NZS 4360 (2004), o padrão IEEE STD 1540-2001 (2001) e o RUP (2003). O PMBOK (A Guide to the Project Management Body of Knowledge) trata do gerenciamento de projetos de uma forma ampla, não sendo específico para software. $\mathrm{O}$ CMMI-SW (Capability Maturity Model Integration for Software) provê um framework para a implantação e melhoria do processo de software das organizações. O RUP (Rational Unified Process) é um processo baseado em melhores práticas de engenharia de software [Reinehr, 2003]. A AS/NZS 4360:2004 é uma norma de âmbito mundial, específica sobre gerenciamento de riscos, desenvolvida na Austrália e Nova Zelândia.

Este artigo analisa cada uma das abordagens de gerenciamento de riscos citadas e propõe um processo para o gerenciamento de riscos em projetos de software, que atenda a todas essas abordagens. Em seguida, apresenta a especialização e implantação do processo em uma organização que atingiu o CMMI-SW nível 3 a partir desse processo.

Este trabalho está organizado como se segue. A seção 2 apresenta um Processo de Gerenciamento de Riscos para projetos de software utilizando várias abordagens de gerenciamento de riscos. A seção 3 mostra a especialização e implantação deste processo. A seção 4 discorre sobre as conclusões deste trabalho.

\section{Processo de Gerenciamento de Riscos para Projetos de Software}

O Processo de Gerenciamento de Riscos proposto baseia-se na área de conhecimento Gerenciamento de Riscos do PMBOK (2004), na área de processo Gerenciamento de Riscos do CMMI-SW (2002), no padrão AS/NZS 4360 (2004), no padrão IEEE Std 1540 (2001), na abordagem de riscos do RUP (2003) e em boas práticas de gerenciamento de riscos da literatura [Boehm, 1991; Farias, 2002; Machado, 2002].

\subsection{Abordagens de Gerenciamento de Riscos}

O PMBOK (2004) - Project Management Body of Knowledge - descreve o conhecimento e as melhores práticas em gerenciamento de projetos, através de nove áreas de conhecimento: Gerência de Integração, Gerência de Escopo, Gerência de Tempo, Gerência de Custo, Gerência de Qualidade, Gerência de Recursos Humanos, Gerência de Comunicação, Gerência de Riscos, e Gerência de Aquisições.

O CMMI-SW (2002) - Capability Maturity Model Integration for Software - foi criado para integrar os diversos modelos do CMM, que atendem as várias atividades relacionadas ao desenvolvimento de software, e ainda torná-lo compatível com a ISO/IEC 15504 (2003). A problemática do risco é abordada nas áreas de processo Planejamento do Projeto, Monitoração e Controle do Projeto e Gerência de Risco. As duas primeiras áreas de processo estão no nível 2 e a última está no nível 3 do CMMISW.

O AS/NZS 4360 (2004) - Australian and New Zealand Standard for Risk Management - é um padrão internacional para o gerenciamento de riscos, que fornece diretrizes para a estruturação e implementação de uma estratégia efetiva para o gerenciamento de riscos. Esse padrão enfatiza que esta estruturação varia de acordo com as necessidades da organização, seus objetivos específicos, seus produtos e 
serviços, bem como as práticas e os processos por ela utilizados. Sua finalidade é estabelecer um framework genérico para o estabelecimento do contexto da gerência de riscos e para a identificação, a análise, a avaliação, o tratamento, a monitoração e a comunicação dos riscos na organização.

O Padrão IEEE Std 1540 - Standard for Software Life Cycle Processes-Risk Management - define um processo contínuo de gerenciamento de riscos aplicável nas disciplinas de engenharia de software e de gerenciamento. É escrito de modo que possa ser aplicado conjuntamente com as normas da série ISO ou independentemente. Este processo consiste nas seguintes atividades: Planejar e Implementar o Gerenciamento de Riscos, Gerenciar o Perfil de Risco do Projeto, Executar a Análise do Risco, Executar o Tratamento do Risco, Executar a Monitoração do Risco e Avaliar o Processo de Gerenciamento de Riscos.

O gerenciamento de riscos no RUP (2003) - Rational Unified Process - é tratado na disciplina de Gerenciamento de Projeto, que se propõe a balancear objetivos concorrentes, gerenciar riscos e restrições, para que a entrega do produto satisfaça seus clientes e usuários. Essa disciplina provê um framework onde o projeto é criado e gerenciado.

O gerenciamento de riscos está integrado ao processo de desenvolvimento, onde as iterações são planejadas com base nos riscos de maior prioridade. Em uma abordagem iterativa, os riscos são mitigados mais cedo, porque os elementos são integrados progressivamente. Além disto, como cada iteração exercita muitos aspectos do projeto, torna-se mais fácil descobrir até que ponto os riscos percebidos estão se materializando, como também descobrir riscos novos [Kroll, 2003].

\subsection{Processo de Gerenciamento de Riscos para Projetos de Software}

Tomando como base as abordagens citadas na seção anterior, foi proposto um Processo de Gerenciamento de Riscos para Projetos de Software [Pereira, 2005]. A Tabela 1 mostra o mapeamento do processo a partir do PMBOK (2004), CMMI-SW (2002), RUP (2003), IEEE Std 1540 (2001) e AS/NZS 4360 (2004). Um dos critérios utilizados para a modelagem das atividades do processo proposto foi que essas atividades fossem comuns à maioria das abordagens utilizadas.

Este processo visa auxiliar no planejamento, na identificação, na análise, no tratamento e na monitoração sistemática dos riscos em projetos de software, contribuindo para a tomada de decisão durante o ciclo de vida do projeto. Esse processo poderá ser especializado para uma determinada organização ou implantado diretamente em projetos de software. A organização poderá ainda optar por adaptar o processo de acordo com as características do projeto, que vier a utilizá-lo. Assim, para projetos de pequeno porte, pode ser executado apenas um subconjunto das atividades do processo proposto, o que deve ser previamente definido no planejamento do gerenciamento de riscos do projeto.

O fluxo principal do Processo de Gerenciamento de Riscos para projetos de software é apresentado na Figura 1, sendo constituído das seguintes macroatividades: (i) Planejar o Gerenciamento de Riscos; (ii) Identificar Riscos, (iii) Analisar Riscos; (iv) Tratar Riscos; (v) Monitorar e Controlar Riscos; e (vi) Avaliar o Processo de Gerenciamento de Riscos. 
Tabela 1. Mapeamento do Gerenciamento de Riscos: PMBOK, CMMI, RUP, IEEE Std 1540, AS/NZS 4360 [Pereira, 2005]

\begin{tabular}{|c|c|c|c|c|c|}
\hline \multirow{2}{*}{\begin{tabular}{c|} 
Processo de \\
Gerenciamento de \\
Riscos para \\
Projetos de \\
Software Proposto \\
(Macroatividades)
\end{tabular}} & PMBOK & CMMI & RUP & \begin{tabular}{|c|} 
Padrão IEEE STD 1540- \\
2001
\end{tabular} & Norma AS/NZS 4360 \\
\hline & $\begin{array}{c}\text { Área de } \\
\text { Conhecimento: } \\
\text { Gerenciamento de } \\
\text { Riscos }\end{array}$ & $\begin{array}{l}\text { Área de Processo: } \\
\text { Gerenciamento de Riscos }\end{array}$ & $\begin{array}{l}\text { Disciplina: } \\
\text { Gerenciamento de Projetos }\end{array}$ & $\begin{array}{l}\text { Padrão específico para o } \\
\text { gerenciamento de riscos }\end{array}$ & $\begin{array}{l}\text { Padrão específico } \\
\text { para o gerenciamento } \\
\text { de riscos }\end{array}$ \\
\hline $\begin{array}{c}\text { Planejar o } \\
\text { Gerenciamento de } \\
\text { Riscos }\end{array}$ & $\begin{array}{l}\text { Planejamento da } \\
\text { Gerência de Riscos }\end{array}$ & $\begin{array}{l}\text { Preparar o Gerenciamento dos Riscos (SG 1): } \\
\text { - Determinar Fontes e Categorias de Riscos (SP 1.1) } \\
\text { - Definir Parâmetros de Riscos (SP 1.2) } \\
\text { - Estabelecer uma Estratégia para o Gerenciamento } \\
\text { de Risco (SP 1.3) }\end{array}$ & $\begin{array}{l}\text { Planejamento do Projeto } \\
\text { - Desenvolver o Plano de } \\
\text { Gerenciamento de Riscos }\end{array}$ & $\begin{array}{l}\text { Planejar e Implementar o } \\
\text { Gerenciamento de Riscos } \\
\text { Gerenciar o Perfil de } \\
\text { Riscos do Projeto }\end{array}$ & $\begin{array}{l}\text { Comunicar e Consultar } \\
\text { Estabelecer o Contexto }\end{array}$ \\
\hline Identificar Riscos & Identificação dos Riscos & $\begin{array}{l}\text { Identificar e Analisar Risco (SG 2) } \\
\text { - Identificar Riscos (SP 2.1) }\end{array}$ & $\begin{array}{l}\text { Avaliar o Escopo do Projeto e } \\
\text { os Riscos } \\
\text { - Identificar e Avaliar os Riscos } \\
\end{array}$ & $\begin{array}{l}\text { Executar a Análise dos } \\
\text { Riscos } \\
\text { - Identificação dos Riscos }\end{array}$ & Identificar Riscos \\
\hline \multirow[t]{2}{*}{ Analisar Riscos } & $\begin{array}{l}\text { Análise Qualitativa dos } \\
\text { Riscos }\end{array}$ & \multirow[t]{2}{*}{$\begin{array}{l}\text { Identificar e Analisar Riscos (SG 2) } \\
\text { - Avaliar, Categorizar e Priorizar Riscos (SP 2.2) }\end{array}$} & \multirow[t]{2}{*}{$\begin{array}{l}\text { Avaliar o Escopo do Projeto e } \\
\text { os Riscos } \\
\text { - Identificar e Avaliar os Riscos }\end{array}$} & \multirow[t]{2}{*}{$\begin{array}{l}\text { Executar a Análise dos } \\
\text { Riscos } \\
\text { - Estimativas dos Riscos } \\
\text { - Avaliação dos Riscos }\end{array}$} & $\begin{array}{l}\text { Analisar Riscos } \\
\text { - Análise Qualitativa } \\
\text { - Análise Semi- } \\
\text { Quantitativa } \\
\text { - Análise-Quantitativa } \\
\end{array}$ \\
\hline & $\begin{array}{c}\text { Análise Quantitativa dos } \\
\text { Riscos }\end{array}$ & & & & Avaliar Riscos \\
\hline Tratar Riscos & $\begin{array}{l}\text { Planejamento das } \\
\text { Respostas aos Riscos }\end{array}$ & $\begin{array}{l}\text { Mitigar Riscos (SG 3) } \\
\text { - Desenvolver Planos de Mitigação de Riscos (SP } \\
\text { 3.1) }\end{array}$ & $\begin{array}{l}\text { Avaliar o Escopo do Projeto e } \\
\text { os Riscos } \\
\text { - Identificar e Avaliar os Riscos } \\
\end{array}$ & $\begin{array}{l}\text { Executar o Tratamento dos } \\
\text { Riscos }\end{array}$ & Tratar Riscos \\
\hline $\begin{array}{c}\text { Monitorar e } \\
\text { Controlar Riscos }\end{array}$ & $\begin{array}{l}\text { Monitoração e Controle } \\
\text { dos Riscos }\end{array}$ & $\begin{array}{l}\text { Mitigar Riscos (SG 3) } \\
\text { - Implementar os Planos de Mitigação de Riscos (SP } \\
\text { 3.2) }\end{array}$ & $\begin{array}{l}\text { Monitorar e Controlar o Projeto } \\
\text { - Monitorar o Status do Projeto }\end{array}$ & $\begin{array}{l}\text { Executar a Monitoração } \\
\text { dos Riscos }\end{array}$ & Monitorar e Revisar \\
\hline $\begin{array}{c}\text { Avaliar o Processo } \\
\text { de Gerenciamento } \\
\text { de Riscos }\end{array}$ & & & & $\begin{array}{l}\text { Avaliar o Processo de } \\
\text { Gerenciamento de Riscos }\end{array}$ & \\
\hline
\end{tabular}


A macroatividade Planejar o Gerenciamento de Riscos deve ser executada no início do projeto e ser repetida se houver mudanças significativas, que justifiquem a necessidade de um novo planejamento. As atividades dos itens (ii), (iii), (iv) e (v) devem ocorrer no mínimo a cada iteração, quando for utilizado o ciclo de vida iterativo e incremental.

A macroatividade Avaliar o Processo de Gerenciamento de Riscos deve ser executada ao final do projeto para averiguar a efetividade do processo e capturar as lições aprendidas.

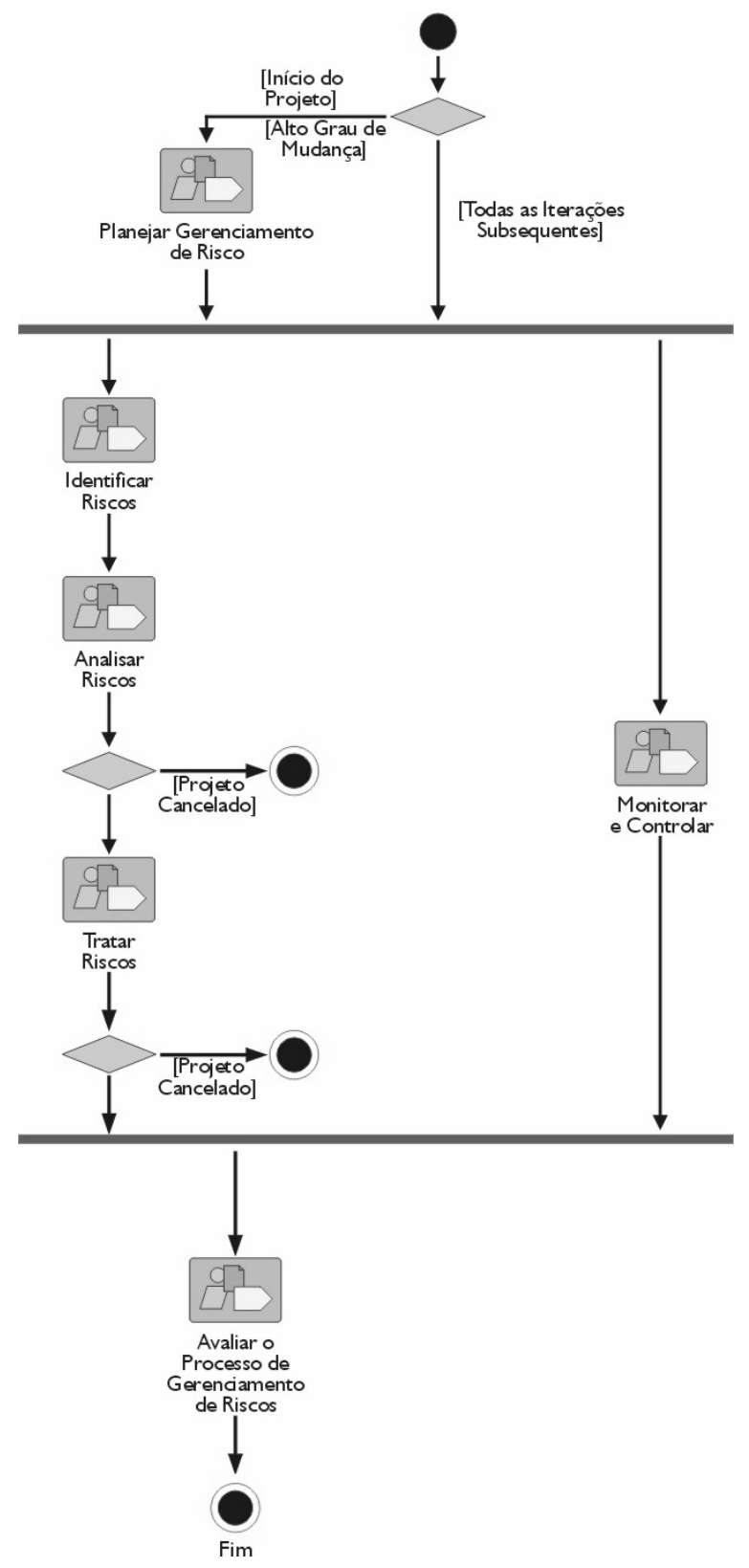

Figura 1. Fluxo do Processo de Gerenciamento de Riscos [Pereira, 2005]

A Tabela 2 apresenta um resumo das macroatividades com suas atividades e seus passos. 
Tabela 2. Detalhamento do Fluxo do Processo de Gerenciamento de Riscos proposto

\begin{tabular}{|c|c|c|}
\hline Macroatividade & Atividades & Passos \\
\hline \multirow{13}{*}{$\begin{array}{l}\text { Planejar o } \\
\text { Gerenciamento de } \\
\text { Riscos }\end{array}$} & \multirow{2}{*}{$\begin{array}{l}\text { Determinar Fontes e } \\
\text { Categorias de Riscos }\end{array}$} & Determinar as Fontes de Riscos \\
\hline & & Determinar as Categorias de Riscos \\
\hline & \multirow{5}{*}{$\begin{array}{l}\text { Definir Parâmetros } \\
\text { para os Riscos }\end{array}$} & Definir Critérios para a Análise dos Riscos \\
\hline & & Definir Critérios o Tratamento dos Riscos \\
\hline & & Definir Métricas \\
\hline & & Definir Limites para cada Categoria de Riscos \\
\hline & & Definir Indicadores para a Avaliação do Processo \\
\hline & \multirow{6}{*}{ Estabelecer Estratégia } & Adaptar o Processo ao Projeto \\
\hline & & Definir Metodologia \\
\hline & & Atribuir Papéis e Responsabilidades \\
\hline & & Definir Periodicidade \\
\hline & & Planejar Comunicação \\
\hline & & Definir a Avaliação do Processo \\
\hline \multirow{5}{*}{ Identificar Riscos } & \multirow{3}{*}{$\begin{array}{l}\text { Identificar Riscos } \\
\text { Potenciais }\end{array}$} & Identificar os Riscos Associados às Fontes e Categorias \\
\hline & & Revisar a Documentação do Projeto \\
\hline & & Revisar as Informações Históricas \\
\hline & \multirow{2}{*}{$\begin{array}{l}\text { Estabelecer o Contexto } \\
\text { dos Riscos }\end{array}$} & Detectar as Circunstâncias ou Condições do Risco \\
\hline & & Identificar Possíveis Conseqüências do Risco \\
\hline \multirow{6}{*}{ Analisar Riscos } & Categorizar os Riscos & Agrupar os Riscos de Acordo com as Categorias \\
\hline & \multirow{3}{*}{$\begin{array}{l}\text { Avaliar Impacto e } \\
\text { Probabilidade }\end{array}$} & Avaliar o Impacto dos Riscos \\
\hline & & Avaliar a Probabilidade de Ocorrência dos Riscos \\
\hline & & Determinar a Exposição ao Risco \\
\hline & Priorizar Riscos & Determinar a Prioridade Relativa dos Riscos \\
\hline & \begin{tabular}{|l|} 
Analisar Riscos \\
Quantitativamente \\
\end{tabular} & Analisar os Riscos Quantitativamente \\
\hline \multirow{9}{*}{ Tratar Riscos } & \multirow{4}{*}{ Selecionar Estratégias } & Definir Limites \\
\hline & & Selecionar Estratégias para Ameaças \\
\hline & & Selecionar Estratégias para Oportunidades \\
\hline & & Selecionar Riscos que Serão Aceitos \\
\hline & \multirow{5}{*}{$\begin{array}{l}\text { Definir e Selecionar } \\
\text { Respostas aos Riscos }\end{array}$} & Definir Ações de Resposta aos Riscos \\
\hline & & Avaliar e Selecionar as Ações \\
\hline & & Definir Plano de Contingências \\
\hline & & Associar Gatilhos aos Riscos \\
\hline & & Designar Proprietários e Envolvidos \\
\hline \multirow{5}{*}{$\begin{array}{l}\text { Monitorar e } \\
\text { Controlar Riscos }\end{array}$} & \multirow{3}{*}{$\begin{array}{l}\text { Monitorar e Controlar } \\
\text { Riscos }\end{array}$} & Acompanhar o Estado Atual dos Riscos \\
\hline & & Executar Plano de Tratamento dos Riscos \\
\hline & & Acompanhar Plano de Tratamento dos Riscos \\
\hline & \multirow{2}{*}{\begin{tabular}{|lll} 
Reportar Estado dos \\
Riscos
\end{tabular}} & Revisar a Documentação dos Riscos \\
\hline & & Comunicar Estado dos Riscos \\
\hline \multirow{3}{*}{$\begin{array}{l}\text { Avaliar o Processo } \\
\text { de Gerenciamento de } \\
\text { Riscos }\end{array}$} & \multirow{3}{*}{ Avaliar o Processo } & Capturar Informações sobre o Processo \\
\hline & & Avaliar e Melhorar o Processo \\
\hline & & Gerar Lições Aprendidas \\
\hline
\end{tabular}


Cada atividade contém um propósito, artefatos de entrada e saída, e papel responsável. Os principais artefatos gerados nesse processo são: o Plano de Gerenciamento de Riscos e o Registro dos Riscos.

Os papéis considerados importantes para a realização das atividades são: o Gerente de Projeto, o Especialista em Riscos e os Envolvidos no Projeto (stakeholder). O Gerente de Projeto é o responsável pela condução do projeto como um todo e participa ou é responsável por grande parte das atividades de gerenciamentos de riscos.

O papel do Especialista em Riscos deve ser desempenhado por um profissional com embasamento em riscos e experiência consolidada em projetos de desenvolvimento de software. O papel do Envolvido no Projeto pode ser desempenhado por qualquer indivíduo que possa ser afetado pelos resultados do projeto.

A seguir será apresentada a especialização do Processo de Gerenciamento de Riscos proposto.

\section{Implantação do Processo de Gestão de Riscos - Estudo de Caso}

O estudo de caso deste trabalho foi realizado no Instituto Atlântico, uma organização de Pesquisa e Desenvolvimento (P\&D), localizada em Fortaleza, Ceará, fundada em novembro de 2001 e avaliada como SW-CMM nível 2 em outubro de 2003. Essa organização atingiu o nível 3 de maturidade do CMMI-SW em fevereiro de 2006.

O Processo de Gestão de Riscos do Instituto Atlântico foi definido e institucionalizado a partir do Processo de Gerenciamento de Riscos proposto, para atender a área de processo de Gerência de Riscos do CMMI-SW nível 3.

O Processo de Gestão de Riscos passou pelas seguintes etapas: (i) Especialização do Processo para a Realidade da Organização; (ii) Implantação em Projetos Piloto e Consultorias; (iii) Institucionalização.

\subsection{Especialização do Processo para a Realidade da Organização}

O processo proposto de gerenciamento de riscos para projetos de software foi especializado para o Instituto Atlântico com o objetivo de institucionalizar a área de processo (PA) de Gerenciamento de Riscos do CMMI-SW.

Inicialmente, foi realizada uma apresentação do processo proposto para um grupo de gerentes de projetos e coordenadores, todos com certificação PMP (Project Management Professional). O objetivo dessa apresentação foi avaliar a adaptabilidade do processo à realidade da organização. A conclusão foi que o processo era totalmente aderente à organização. No entanto, deveria ser feita uma especialização desse processo de acordo com os padrões da organização, utilizando seu framework de processos. O resultado dessa especialização é apresentado na Figura 2.

A organização decidiu pela não criação do papel do Especialista em Riscos, apesar do reconhecimento de sua importância para as atividades de gerenciamento de riscos. Esse papel foi incorporado às atividades de coordenadores externos ao projeto. Foi determinada a utilização dos papéis já previamente definidos no Processo de Gestão de Projetos da organização, no processo de gestão de riscos especializado. São eles: Gerente, Coordenador, e Analista. 


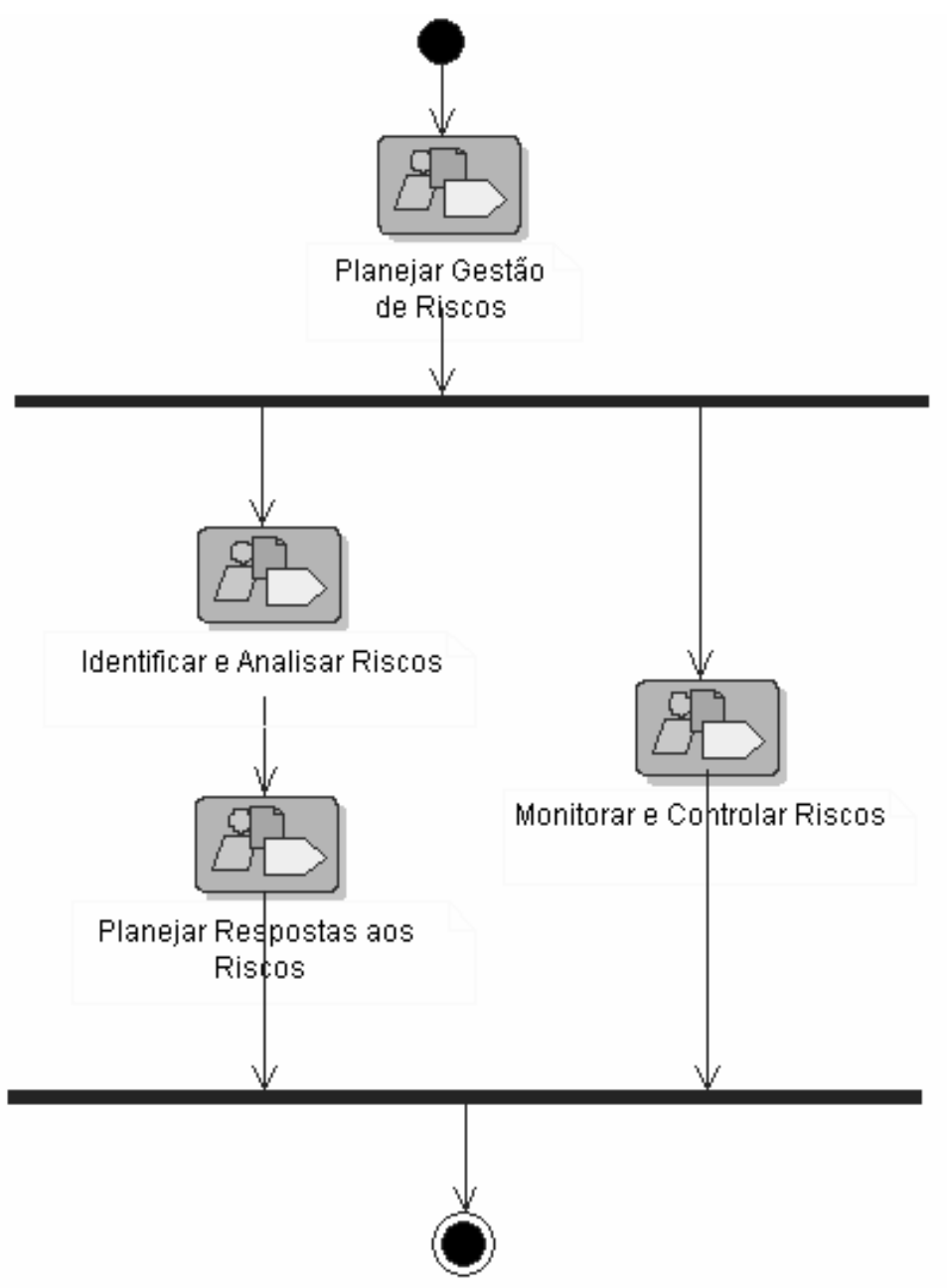

Figura 2. Fluxo do Processo de Gestão de Riscos Especializado

Os artefatos do processo proposto também deveriam ser substituídos pelos artefatos correspondentes já existentes no repositório de processos da organização. Apenas seriam elaborados os artefatos específicos de gerenciamento de riscos. Sendo assim, foram criados o Plano de Gestão de Riscos e a Lista de Riscos.

Durante a definição do processo, foram realizadas várias entrevistas com gerentes de projetos e coordenadores, para a obtenção de informações sobre as práticas de gerenciamento de riscos utilizadas no dia a dia dos projetos e sobre as dificuldades encontradas.

Antes da implantação do CMMI nível 3, apesar de não haver um processo definido, a organização já utilizava as práticas de identificação, atribuição de valores à probabilidade e ao impacto dos riscos identificados, planejamento de respostas e acompanhamento de riscos. No entanto, a exposição ao risco não era utilizada para a priorização dos riscos e não havia critérios definidos para a seleção dos riscos que seriam tratados. 
O coordenador do projeto tentava acompanhar todos os riscos ou mesmo apenas os identificava no início do projeto e depois reagia a eles, quando constatava que um risco havia ocorrido, através da execução de um plano de contingências. $O$ acompanhamento pouco efetivo dos riscos e das ações definidas para seu tratamento foi uma das principais falhas apontadas pelos coordenadores em seus projetos.

Entre outras dificuldades no gerenciamento de riscos, podemos citar:

- Acompanhamento dos riscos;

- Acompanhamento das ações no cronograma;

- Definição de quando a ação deveria ocorrer;

- Como verificar se a estratégia de mitigação estaria sendo efetiva;

- Ausência de uma base histórica dos riscos mais comuns;

- A ferramenta utilizada não facilitava o acompanhamento dos riscos.

As práticas de gerenciamento de riscos faziam parte do processo de Gestão de Projetos. As práticas de identificar, analisar, priorizar, associar ações de mitigação e contingência e acompanhar riscos eram mencionadas nos procedimentos institucionais de Planejamento de projetos e Acompanhamento de projetos. Esta forma de implementação atendia apenas às exigências do nível 2 do SW-CMM.

O passo seguinte foi a elaboração dos fluxos do Processo de Gestão de Riscos especializado, utilizando o modelo de definição de processos e os papéis existentes para os processos da organização. A organização optou por fluxos mais simplificados e um maior detalhamento nos procedimentos descritos para cada atividade. O fluxo principal adaptado e os fluxos das macroatividades foram submetidos à aprovação.

O Processo de Gestão de Riscos (especializado) foi institucionalizado e apresentado à equipe do EPG (Engineering Process Group) e a uma consultoria externa de CMMI. Foram feitas apenas algumas observações e sugestões com o intuito de melhorar o processo especializado, uma vez que este já estava aderente ao CMMI-SW, segundo essa consultoria.

Em seguida, foram definidas as categorias de riscos a serem utilizadas pelos projetos. Foi realizado um levantamento das fontes de riscos mais comuns nos projetos da organização, sendo elaborado um documento de orientação para o levantamento dos riscos baseados nas fontes e categorias de riscos identificadas.

A Tabela 3 apresenta o mapeamento das macroatividades do Processo de Gerenciamento de Riscos (proposto) com os artefatos gerados e os processos (procedimentos) utilizados pelas macroatividades do Processo de Gestão de Riscos (especializado) da organização. 
Tabela 3. Mapeamento do Processo Proposto com o Processo Especializado

\begin{tabular}{|c|c|c|}
\hline \multicolumn{2}{|c|}{$\begin{array}{c}\text { Processo de Gerenciamento de Riscos } \\
\text { (Proposto) }\end{array}$} & \multirow{2}{*}{$\begin{array}{l}\text { Processo de Gestão de Riscos } \\
\text { (Especializado) }\end{array}$} \\
\hline Macroatividades & Atividades & \\
\hline \multirow{9}{*}{$\begin{array}{l}\text { Planejar o } \\
\text { Gerenciamento } \\
\text { de Riscos }\end{array}$} & \multirow{2}{*}{$\begin{array}{l}\text { Determinar Fontes e } \\
\text { Categorias de Riscos }\end{array}$} & - Documento de Orientação detalhando fontes categorias de riscos \\
\hline & & - $\quad$ Ferramenta de Gestão de Riscos \\
\hline & \multirow{4}{*}{$\begin{array}{l}\text { Definir Parâmetros } \\
\text { para os Riscos }\end{array}$} & - $\quad$ Procedimento Analisar Riscos \\
\hline & & - $\quad$ Procedimento Planejar Respostas aos Riscos \\
\hline & & - $\quad$ Processo de Medição e Análise \\
\hline & & - $\quad$ Ferramenta de Gestão de Riscos \\
\hline & \multirow{3}{*}{$\begin{array}{l}\text { Estabelecer } \\
\text { Estratégia }\end{array}$} & - $\quad$ Processo de Gestão de Projetos \\
\hline & & - $\quad$ Elaborar Plano de Gestão de Riscos \\
\hline & & - $\quad$ Processo de Garantia da Qualidade \\
\hline \multirow{5}{*}{ Identificar Riscos } & \multirow{4}{*}{$\begin{array}{l}\text { Identificar Riscos } \\
\text { Potenciais }\end{array}$} & - $\quad$ Procedimento Identificar Riscos \\
\hline & & - $\quad$ Procedimento Elaborar Plano de Gestão de Riscos \\
\hline & & - $\quad$ Documento Orientação - Técnicas de Identificação dos Riscos \\
\hline & & - $\quad$ Elaborar Plano de Gestão de Riscos \\
\hline & $\begin{array}{l}\text { Estabelecer o } \\
\text { Contexto dos Riscos }\end{array}$ & - $\quad$ Procedimento Identificar Riscos \\
\hline \multirow{4}{*}{ Analisar Riscos } & Categorizar Riscos & - $\quad$ Procedimento Identificar Riscos \\
\hline & $\begin{array}{l}\text { Avaliar Impacto e } \\
\text { Probabilidade }\end{array}$ & - $\quad$ Procedimento Analisar Riscos \\
\hline & Priorizar Riscos & - $\quad$ Procedimento Analisar Riscos \\
\hline & $\begin{array}{l}\text { Analisar Riscos } \\
\text { Quantitativamente }\end{array}$ & - $\quad$ Esta atividade não será realizada inicialmente \\
\hline \multirow[t]{2}{*}{ Tratar Riscos } & $\begin{array}{l}\text { Selecionar } \\
\text { Estratégias }\end{array}$ & $\begin{array}{ll}\text { - } & \text { Procedimento Planejar Respostas aos Riscos } \\
\text { - } & \text { (Riscos positivos não serão tratados inicialmente) }\end{array}$ \\
\hline & $\begin{array}{l}\text { Definir e Selecionar } \\
\text { Respostas aos Riscos } \\
\end{array}$ & - $\quad$ Procedimento Planejar Respostas aos Riscos \\
\hline \multirow{2}{*}{$\begin{array}{l}\text { Monitorar e } \\
\text { Controlar Riscos }\end{array}$} & $\begin{array}{l}\text { Monitorar e } \\
\text { Controlar Riscos }\end{array}$ & - $\quad$ Procedimento Monitorar e Controlar Riscos \\
\hline & $\begin{array}{l}\text { Reportar Estado dos } \\
\text { Riscos }\end{array}$ & - $\quad$ Procedimento Reportar Estado dos Riscos \\
\hline $\begin{array}{l}\text { Avaliar o Processo } \\
\text { de Gerenciamento } \\
\text { de Riscos }\end{array}$ & Avaliar o Processo & - $\quad$ Processo de Gestão de Processos \\
\hline
\end{tabular}

\subsection{Implantação em Projetos Piloto e Consultorias}

Inicialmente, foram escolhidos quatro projetos piloto para a implantação do processo. Em seguida, os gerentes e coordenadores dos projetos escolhidos foram orientados na execução do Processo de Gestão de Riscos especializado.

Para os quatro projetos piloto selecionados foi realizado um mentoring de utilização do Processo de Gestão de Riscos. Nesses projetos, a equipe do EPG monitorava diariamente a utilização do processo e fornecia orientações para solução das não conformidades identificadas. Com base na experiência obtida nesses projetos piloto, foram tomadas algumas ações como as que se seguem: 
- Divulgação de documento contendo as fontes de risco mais comuns da organização;

- Inserção dessas fontes na ferramenta de riscos do projeto;

- Revisão dos parâmetros utilizados para a análise dos riscos;

- Explicação mais detalhada de cada uma das estratégias de tratamento dos riscos no procedimento;

- Maior ênfase na importância da definição de ações para responder aos riscos;

- Maior ênfase na importância da definição de gatilhos para viabilizar o acompanhamento dos riscos.

A implantação do Processo de Gestão de Riscos nos projetos pilotos foi uma etapa de fundamental importância para a realização dos ajustes necessários e estabilização desse processo.

Durante esta etapa, a organização foi submetida à avaliação Classe C. O plano de ação resultante dessa avaliação considerou o Processo de Gestão de Riscos (especializado) com estando totalmente definido, tendo sido registrada apenas uma lacuna, referente ao acompanhamento dos riscos de baixa severidade. O texto do procedimento foi melhorado, tornando-o mais claro, solucionando assim, a lacuna identificada.

Foram também registradas algumas recomendações referentes a uma melhor definição das fronteiras dos riscos e da utilização de gatilhos, para um acompanhamento mais efetivo dos riscos e de suas ações. A implantação dessas recomendações foi viabilizada pela substituição da ferramenta de gestão de riscos utilizada.

Assim sendo, como resultado da utilização deste processo de gestão de riscos, as lições aprendidas nos projetos pilotos foram incluídas nesse processo e o mesmo passou posteriormente pelas avaliações Classe B, Avaliação Interna e Classe A, todas utilizando o SCAMPI (2001) - Standard CMMI Assessment Method for Process Improvement. Na avaliação Classe $\mathrm{B}$, foi necessário apenas um pequeno ajuste na utilização desse processo em um dos projetos avaliados (quatro projetos foram escolhidos para a avaliação). Na Avaliação Interna não foi identificada nenhuma nãoconformidade e a avaliação Classe A certificou o nível de maturidade 3 CMMI à organização sem detectar nenhum problema.

\subsection{Institucionalização do Processo de Gestão de Riscos}

A disseminação do conhecimento sobre o Processo de Gestão de Riscos de software foi realizada para os principais envolvidos nesse processo, através de treinamentos planejados de acordo com o Programa de Treinamento Organizacional, de modo a garantir que tivessem o conhecimento e as habilidades necessárias para desempenharem seus papéis. Como complemento, os membros da equipe de QA's (Quality Assurance) tiveram papel fundamental, orientando o seguimento do processo e participando na execução de algumas atividades.

Com o processo estável e os principais envolvidos treinados, a institucionalização do Processo de Gestão de Riscos em toda a organização ocorreu naturalmente. Os membros das equipes dos projetos sentiam-se motivados para a execução desse processo, pois compreendiam a sua importância. 
Atualmente, doze projetos já utilizam o Processo de Gestão de Riscos especializado. Destes, foram selecionados quatro projetos para demonstração dos resultados obtidos pela aplicação desse processo. Esses projetos por questões de confidencialidade serão denominados de Projeto A, Projeto B, Projeto C, e Projeto D.

Serão apresentadas as características gerais de cada projeto e dos riscos identificados em cada um deles. Para cada projeto, será apresentada uma tabela contendo o percentual total dos riscos identificados por status e distribuídos de acordo com seu grau de severidade.

\subsubsection{Projeto $A$}

O Projeto A tem como objetivo migrar um sistema existente para uma arquitetura distribuída. Utiliza a plataforma J2EE (Java 2 Platform, Enterprise Edition) e o ambiente de desenvolvimento Eclipse. Esse projeto é de médio porte e o tamanho da equipe alocada é de 11 pessoas.

Para este projeto foram identificados 15 riscos, sendo os principais riscos referentes ao fato de o desenvolvimento estar sendo realizado entre a organização e o próprio cliente, além do pouco conhecimento dos módulos desse projeto.

Alguns dos resultados do acompanhamento dos riscos foram a percepção de que novos riscos surgiram com o maior conhecimento do projeto, e que a efetivação de um risco referente a recursos humanos teve impacto na análise e projeto. A Tabela 3 demonstra uma visão geral dos riscos do projeto em março de 2006.

Tabela 3. Riscos do Projeto $A$

\begin{tabular}{|c|c|c|c|c|}
\hline \multicolumn{5}{|c|}{ Projeto $A$} \\
\hline \multirow{2}{*}{ Status } & \% status & \multicolumn{3}{|c|}{ Severidade } \\
\cline { 3 - 5 } & de Riscos & Baixa & Média & Alta \\
\hline Ativo & $53,3 \%$ & $6,7 \%$ & $20,0 \%$ & $26,7 \%$ \\
\hline Efetivado & $33,3 \%$ & $0,0 \%$ & $13,3 \%$ & $20,0 \%$ \\
\hline Transferido & $0,0 \%$ & $0,0 \%$ & $0,0 \%$ & $0,0 \%$ \\
\hline Eliminado & $13,3 \%$ & $0,0 \%$ & $13,3 \%$ & $0,0 \%$ \\
\hline Total & $\mathbf{1 0 0} \%$ & $\mathbf{6 , 7} \%$ & $\mathbf{4 6 , 7 \%}$ & $\mathbf{4 6 , 7 \%}$ \\
\hline
\end{tabular}

\subsubsection{Projeto $B$}

O Projeto $B$ tem como objetivo o desenvolvimento de um sistema corporativo para a área financeira, utilizando a plataforma J2EE e o ambiente de desenvolvimento Eclipse. O ciclo de vida utilizado nesse projeto é o iterativo-incremental e o sistema é modelado em UML (Unified Modeling Language). Este projeto é de grande porte e o tamanho da equipe alocada é de 45 pessoas.

Para este projeto foram identificados 45 riscos, sendo os principais riscos referentes à integração com outros fornecedores, perfil do usuário, substituição de legado e porte do projeto.

Alguns dos resultados do acompanhamento dos riscos referem-se à necessidade de definição de ações de mitigação ou eliminação mais efetivas. A Tabela 4 apresenta uma visão geral dos riscos do projeto em fevereiro de 2006.

\section{Tabela 4. Riscos do Projeto B}




\begin{tabular}{|c|c|c|c|c|}
\hline \multicolumn{5}{|c|}{ Projeto B } \\
\hline \multirow{2}{*}{ Status } & \% status & \multicolumn{3}{|c|}{ Severidade } \\
\cline { 3 - 5 } & de Riscos & Baixa & Média & Alta \\
\hline Ativo & $50,0 \%$ & $0,0 \%$ & $25,0 \%$ & $25,0 \%$ \\
\hline Efetivado & $0,0 \%$ & $0,0 \%$ & $0,0 \%$ & $0,0 \%$ \\
\hline Transferido & $0,0 \%$ & $0,0 \%$ & $0,0 \%$ & $0,0 \%$ \\
\hline Eliminado & $50,0 \%$ & $25,0 \%$ & $25,0 \%$ & $0,0 \%$ \\
\hline Total & $100 \%$ & $25,0 \%$ & $50,0 \%$ & $25,0 \%$ \\
\hline
\end{tabular}

\subsubsection{Projeto $C$}

O Projeto $C$ tem como objetivo desenvolver dois produtos de software para a indústria de telecomunicações. Utiliza a plataforma J2ME (Java 2 Platform, Micro Edition) e o ambiente de desenvolvimento Eclipse. Esse projeto é de pequeno porte e o tamanho da equipe alocada é de 6 pessoas.

Para esse projeto foram identificados apenas 4 riscos, em decorrência do porte do projeto. O principal risco refere-se à necessidade de alocação dos recursos do projeto para atividades externas ao projeto. A Tabela 5 demonstra uma visão geral dos riscos do projeto em novembro de 2005.

Tabela 5. Riscos do Projeto C

\begin{tabular}{|c|c|c|c|c|}
\hline \multicolumn{5}{|c|}{ Projeto $C$} \\
\hline \multirow{2}{*}{ Status } & \% status & \multicolumn{3}{|c|}{ Severidade } \\
\cline { 3 - 5 } & de Riscos & Baixa & Média & Alta \\
\hline Ativo & $74,3 \%$ & $2,9 \%$ & $28,6 \%$ & $42,9 \%$ \\
\hline Efetivado & $\mathbf{8 , 6 \%}$ & $\mathbf{0 , 0} \%$ & $\mathbf{0 , 0} \%$ & $\mathbf{8 , 6 \%}$ \\
\hline Transferido & $\mathbf{0 , 0} \%$ & $\mathbf{0 , 0} \%$ & $\mathbf{0 , 0} \%$ & $\mathbf{0 , 0} \%$ \\
\hline Eliminado & $\mathbf{1 7 , 1} \%$ & $2,9 \%$ & $2,9 \%$ & $11,4 \%$ \\
\hline Total & $100 \%$ & $\mathbf{6 , 7} \%$ & $31,4 \%$ & $\mathbf{6 2 , 9 \%}$ \\
\hline
\end{tabular}

\subsubsection{Projeto $D$}

O Projeto $D$ tem como objetivo o desenvolvimento de um sistema corporativo de controle de processos, em uma interface Web na plataforma J2EE, utilizando as tecnologias HTML, Javascript, Struts Tiles, Servlets / JSP, XML, JBoss, MSSQL e EJB (Enterprise Java Beans). Este projeto é de médio porte e o tamanho da equipe alocada é de 24 pessoas.

Para esse projeto foram identificados 16 riscos, sendo os principais referentes à distância do cliente e à necessidade de desenvolvimento de novos módulos, com suporte aos módulos implantados e em produção. Alguns dos resultados do acompanhamento dos riscos foram a percepção de que o projeto apresenta um volume considerável de riscos com severidade média e alta e que $75 \%$ dos riscos estão ativos. A Tabela 6 exibe uma visão geral dos riscos do projeto em novembro de 2005. 
Tabela 6. Riscos do Projeto D

\begin{tabular}{|c|c|c|c|c|}
\hline \multicolumn{5}{|c|}{ Projeto $D$} \\
\hline \multirow{2}{*}{ Status } & \% status & \multicolumn{3}{|c|}{ Severidade } \\
\cline { 3 - 5 } & de Riscos & Baixa & Média & Alta \\
\hline Ativo & $75,0 \%$ & $6,3 \%$ & $12,5 \%$ & $56,3 \%$ \\
\hline Efetivado & $12,5 \%$ & $6,3 \%$ & $\mathbf{0 , 0} \%$ & $6,3 \%$ \\
\hline Transferido & $\mathbf{6 , 3 \%}$ & $\mathbf{0 , 0} \%$ & $\mathbf{0 , 0} \%$ & $\mathbf{6 , 3 \%}$ \\
\hline Eliminado & $\mathbf{6 , 3 \%}$ & $\mathbf{0 , 0} \%$ & $\mathbf{0 , 0} \%$ & $\mathbf{6 , 3 \%}$ \\
\hline Total & $100 \%$ & $12,5 \%$ & $12,5 \%$ & $75,0 \%$ \\
\hline
\end{tabular}

\section{Conclusões}

O Processo de gerenciamento de riscos para projetos de software, proposto neste trabalho, trouxe as seguintes contribuições:

- Destacar a importância da gerência de riscos para projetos de software, agregando em um só processo, os fundamentos de modelos de riscos reconhecidos pela comunidade científica, e que são amplamente utilizados;

- Atender à área de processo Gerência de Riscos do CMMI-SW, nível 3, as exigências do PMBOK (2004), as práticas de gerenciamento de riscos do RUP (2003), ao padrão IEEE STD 1540-2001 (2001) e as orientações da norma AS/NZS 4360 (2004), adequadas ao desenvolvimento de software.

- Formalizar um processo de gerenciamento de riscos para projetos de software em geral, sendo também aderente ao ciclo de vida iterativo e incremental;

- Contribuir para que o gerenciamento de riscos em projetos de software seja mais efetivo, resultando em um desenvolvimento de software com qualidade.

Com a experiência de especialização do processo proposto em uma organização, certificada CMMI-SW nível 3 em fevereiro de 2006, tivemos as seguintes lições aprendidas:

- Por mais completo que seja um processo, é importante que ele seja adaptado à realidade e necessidades da organização. Levar a empresa simplesmente a adaptar-se ao processo, sem considerar suas peculiaridades, pode gerar um esforço desnecessário e que, via de regra, resulta em insatisfação dos usuários;

- O fato de ter um processo preliminar que atendesse ao modelo CMMI-SW facilitou a definição do processo de gerenciamento de risco na organização;

- Ter gerentes e coordenadores certificados como PMP's (Project Management Professional) facilitou bastante o entendimento e a implantação do processo, pois estes já possuíam conhecimento em gerenciamento de projetos, havendo uma motivação adicional em colocar seu aprendizado em prática;

- A implantação de um processo em projetos pilotos facilita de sobremaneira a institucionalização do processo, evitando assim que possíveis problemas possam vir a ser propagados por toda a organização, podendo gerar descrédito do próprio processo; 
- O apoio de uma consultoria especializada foi fundamental para tornar o processo eficaz e otimizado.

Como próximos passos, a organização pretende fazer uso do conceito de riscos positivos ou oportunidades, assim como as estratégias para potencializar seus efeitos para projetos de desenvolvimento de software.

Como perspectivas futuras com relação a este trabalho, vislumbra-se a elaboração de uma base histórica que dê suporte às atividades de gerenciamento de riscos da organização. Essa base histórica poderá permitir a definição de parâmetros mais acurados para o gerenciamento de riscos, de acordo com as categorias de riscos adotadas pela organização, a seleção de respostas aos riscos mais eficazes, e favorecer a melhoria contínua desse processo.

\section{Referências}

AS/NZS 4360 (2004) “Australian/New Zealand Standard for Risk Management”.

Boehm, B. W. Software Risk Management: Principles and Practices, IEEE Software. V. 8. N. 1. p. 32-41, Jan. 1991.

Brown, N. (1996) “Industrial - Strength Management Strategie, IEEE”.

CMMI Product Team. (2002) "CMMI for Systems Engineering/Software Engineering”, Version 1.1 Staged Representation (CMU/SEI-2002-TR-029, ESC-TR-2002-029). Pittsburgh, PA: Software Engineering Institute, Carnegie Mellon University.

Gusmão, C. M. G., Moura, P. H. (2003) "ISO, CMMI, and PMBOK Risk Management: a Comparative Analysis”. The International Journal of Applied Management and Technology, Volume 1, Number 1.

IEEE Std 1540. (2001) “Standard for Software Life Cycle Processes-Risk Management”.

ISO/IEC 15504. (2003) “Software Process Assessment”.

Kroll, Per \& Kruchten, Philippe. (2003) "The Rational Unified Process made easy: a practitioner's guide to the RUP”, Pearson Education.,

Machado, C. A. F. (2002) “A-Risk: Um Método para Identificar e Quantificar Risco de Prazo de Projetos de Desenvolvimento de Software”, Dissertação de Mestrado, PUC-PR, Curitiba.

Pereira, P. C. R. (2005) “Um Processo de Gerenciamento de Riscos para Projetos de Software”, Dissertação de Mestrado, Universidade de Fortaleza (Unifor), Novembro, 2005.

PMBOK. (2004) PMI Standards Committee. “A Guide to the Project Management Body of Knowledge”, Third Edition, PMI Publishing Division, Philadelphia, USA.

Reinehr, S.S., Balduino, R., Machado, C. A. F., Pessoa, M. S. (2003) "Implementing ISO/IEC 12207 Standard using Rational Unified Process”. Software Engineering Research and Practice.

RUP. (2003) "Rational Unified Process”, Version 2003.06.00.65, CD-ROM. Rational Software Corporation, Cupertino, California.

SCAMPI. (2001) Standard CMMI ${ }^{\mathrm{SM}}$ Appraisal Method for Process Improvement (SCAMPI $^{\mathrm{SM}}$ ), Version 1.1: Method Definition Document. CMU/SEI-2001-HB-001.

Sommerville, Ian. (2003) “Engenharia de software”. 7. ed. São Paulo: Addison Wesley. 